\title{
Video-assisted thoracoscopic surgery for the central bronchogenic carcinoma with invasion of the main bronchus and pulmonary artery: A case report
}

\author{
JUN WAN and RENQUAN ZHANG
}

Department of Thoracic Surgery, The First Affiliated Hospital of Anhui Medical University, Hefei, Anhui 230022, P.R. China

Received June 15, 2016; Accepted August 12, 2016

DOI: $10.3892 / \mathrm{mco} .2016 .1016$

\begin{abstract}
Surgical treatment for central bronchogenic carcinoma with invasion of the main bronchus and pulmonary artery often include bronchial anastomosis and pulmonary angioplasty. As a technique, video-assisted thoracoscopic surgery (VATS) lobectomy has proven to reduce the rate of occurrence of complications, and thereby obtain improved survival rates. In the present case study, its use in treating central bronchogenic carcinoma with invasion of the main bronchus and pulmonary artery is reported. A case study of a 68-year-old man with a history of smoking 15-20 cigarettes per day for 40 years is described, who presented with a cough and hemoptysis. A bronchial sleeve left upper lobectomy and pulmonary angioplasty were performed with complete VATS and routine lymph node dissection. The preoperative diagnosis of squamous cell carcinoma (SCC) of the lung (cT2, N2, M0, stage IIIA) was confirmed as SCC through the postoperative pathological examination, leading to the tumor staging: pT2, N1, M0, stage IIB. These results were obtained without sacrificing the oncological principles of thoracic surgery. It has been demonstrated that VATS may be applied for treating central bronchogenic carcinoma with invasion of the main bronchus and pulmonary artery. However, end-to-end bronchial anastomosis and continuous suture of the pulmonary artery were difficult to perform, and use of the VATS procedure is on a learning curve, which will be informative for surgeons and their assistants.
\end{abstract}

\section{Introduction}

Central bronchogenic carcinoma is usually accompanied with cancerous invasion of the main bronchus and pulmonary

Correspondence to: Dr Renquan Zhang, Department of Thoracic Surgery, The First Affiliated Hospital of Anhui Medical University, 218 Jixi Road, Hefei, Anhui 230022, P.R. China

E-mail: wanjun622@126.com

Key words: video-assisted thoracoscopic surgery, central bronchogenic carcinoma, bronchial anastomosis, pulmonary angioplasty artery, and therefore the surgical operation to be performed requires bronchial anastomosis and pulmonary angioplasty, which are performed by traditional thoracotomy.

Video-assisted thoracoscopic surgery (VATS) is an emerging technology in minimally invasive surgery, which provides painless surgery with a short recovery time. Bronchial anastomosis and pulmonary angioplasty may be completed using VATS. The worldwide experience with VATS lobectomy is now sufficiently robust to enable a comparison of this procedure to be made with traditional thoracotomy. In all five lobes of the lung, the stage of the operation that has the greatest difficulty is VATS sleeve left upper lobectomy for the special anatomical structure. In the present case report, and application of VATS for the treatment of central lung cancer of the upper lobe with invasion of the main bronchus and pulmonary artery is presented.

\section{Case report}

All procedures used in the present case study were approved by the Clinical Medical Research Ethics Committee of the First Affiliated Hospital of Anhui Medical University (Hefei, China).

A 68-year-old man whose smoking history was 15-20 cigarettes per day for 40 years presented with a cough and hemoptysis. In November 2011, the patient was found to have an abnormal shadow in the left pulmonary hilum, based on a chest X-ray (Fig. 1A), and so the patient was referred to our hospital (The First Affiliated Hospital of Anhui Medical University, Hefei, China) for further examination and therapy. Physical examination revealed that there were no palpable superficial lymph nodes. Blood chemistry data were unremarkable, with the exception of a decreased level of total protein (TPR) of $56.1 \mathrm{~g} / 1$ (normal range, 60.0-80.0 g/l) and a slightly increased level of total bilirubin to $19.0 \mu \mathrm{mol} / \mathrm{l}$ (normal range, 5.1-19.0 $\mu \mathrm{mol} / \mathrm{l}$ ). The tumor markers for squamous cell carcinoma (SCC) antigen (normal range, 0-2 ng/ml), sialyl SSEA-1 antigen (SLX; normal range, 0-38 U/ml), and neuron-specific enolase (NSE; normal range, $0-10 \mathrm{ng} / \mathrm{ml}$ ) were slightly increased to $2.6 \mathrm{ng} / \mathrm{ml}, 44.3 \mathrm{U} / \mathrm{ml}$ and $10.12 \mathrm{ng} / \mathrm{ml}$, respectively. Bronchoscopy revealed an occlusion in the distal portion of the left main bronchus by a cauliflower-like mass (Fig. 1B). An enhanced computed tomography (CT) scan (Siemens SOMATOM Definition AS 64-slice computed 
A

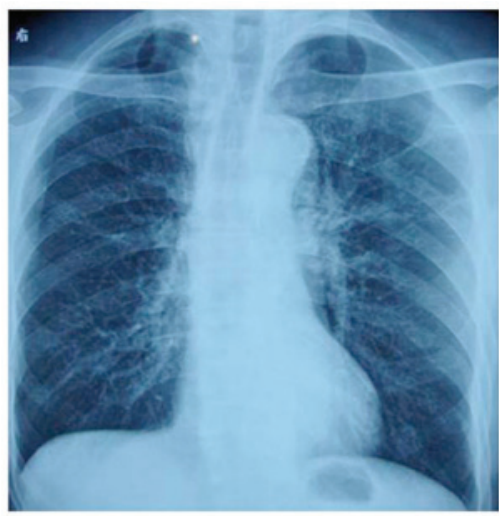

C

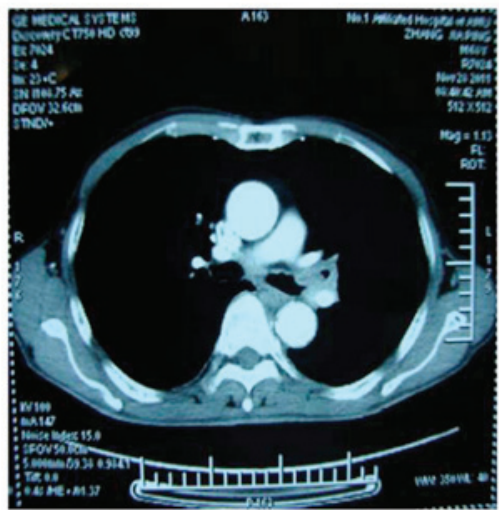

B

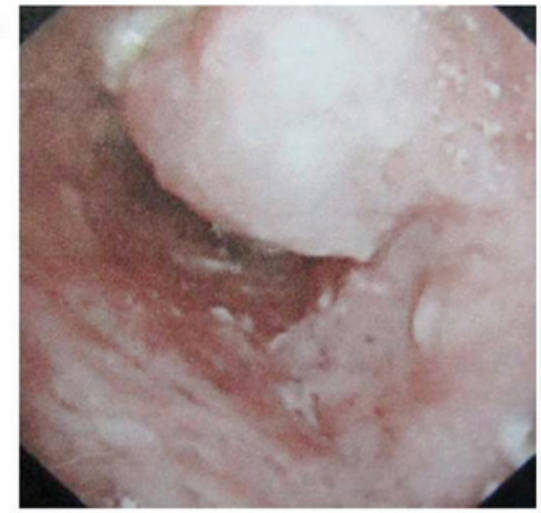

D

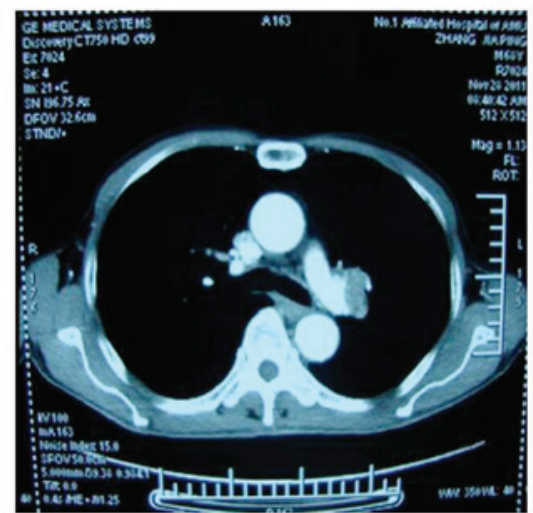

Figure 1. Preoperative examination of the patient. (A) The chest X-ray demonstrated a shadow of a mass in the left pulmonary hilum. (B) Bronchoscopy revealed a cauliflower-like mass which blocked the distal portion of the left main bronchus. (C) Chest computed tomography demonstrated a well-defined mass located on the left upper lobe bronchus. (D) Chest computed tomography demonstrated that the mass had pulmonary artery invasion.

tomography system; Siemens Healthineers, Erlangen, Germany) revealed a well-defined mass of soft tissue, with a coarse edge measuring $3.8 \times 4.2 \times 4.0 \mathrm{~cm}$, in the upper lobe of the left lung close to the hilum, and enlarged mediastinal lymph nodes were also identified. The mass appeared to be located on the left upper lobe bronchus, and have pulmonary artery invasion (Fig. 1C and D). Pulmonary function tests revealed a moderate obstructive ventilatory disorder and severe abnormality of small airway function. Considering all the results collectively, the preoperative stage of the tumor was assessed to be cT2, N2, M0, IIIA, and the patient was proposed for a VATS lobectomy.

The patient was placed in the right-lateral decubitus position. A $3.5 \mathrm{~cm}$ incision was made as the main operating hole in the third intercostal space of the anterior axillary line position. A thoracoscopic observation hole was made using a $12 \mathrm{~cm}$ trocar in the eighth intercostal space of the midaxillary line. In addition, a $3.5 \mathrm{~cm}$ incision was made as the operational assist hole, and a further $12 \mathrm{~cm}$ trocar was placed in the fifth intercostal space of the posterior axillary line position. A complete fissure was identified. Digital palpation confirmed the presence of a mass in the left upper lobe. The instruments were inserted to start the dissection of the pulmonary ligament and mediastinal pleura, and the superior pulmonary vein was exposed and resected using a $45 \mathrm{~mm}$ white linear stapler (Fig. 2A). Amputation of the pulmonary fissure was performed using an endoscopic linear cutter scalpel (Endo-GIA ${ }^{\mathrm{TM}}$; Covidien LLC, Mansfield, MA, USA), and it was possible to observe that the carcinoma located on the left main bronchus had partly invaded the left pulmonary artery. After having cut and freed the left main bronchus, the left pulmonary artery was freed and blocked (Fig. 2B). The left upper lobe was removed in a protective bag, and a systematic lymph node dissection was completed.

The left pulmonary artery was repaired using a reciprocating continuous 4-0 Prolene ${ }^{\circledR}$ suture (Fig. 2C). The proximal occlusion clamp was opened, and subsequently the telecentric end occlusion clamp was opened to make the pulmonary perfusion. After the inferior pulmonary ligament had been divided to release the tension of airway anastomosis, end-to-end anastomosis was initiated. The midpoint of the offside wall of the bronchus was chosen as the starting point, then a 3-0 Prolene ${ }^{\circledR}$ continuous suture was used to close the membranous and bronchial cartilage from the posterior to the anterior end, dragging them tight simultaneously with the help of an endoscopic knot-pusher. Following the completion of all the procedures, the left lung was inflated, and air leakage did not occur.

The intraoperative bleeding volume was $120 \mathrm{ml}$, and no intraoperative complications occurred. The pathological examination revealed a $2.6 \times 1.7 \times 2.7 \mathrm{~cm}$ solid carcinoma, which the resultant microscopic examination identified as SCC (Fig. 3) with one hilar lymph node involvement (a total of seven lymph nodes were studied), leading us to consider that the postoperative stage was pT2, N1, M0, IIB. Thoracic drainage tubes were removed on the eighth postoperative day, and the patient was discharged from the hospital on the ninth postoperative day. 
A

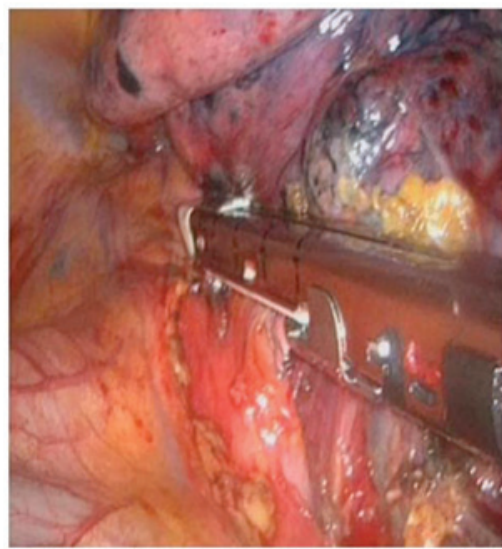

C

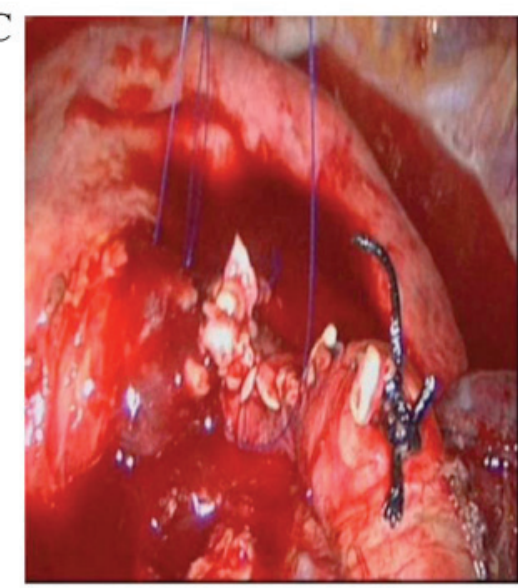

B

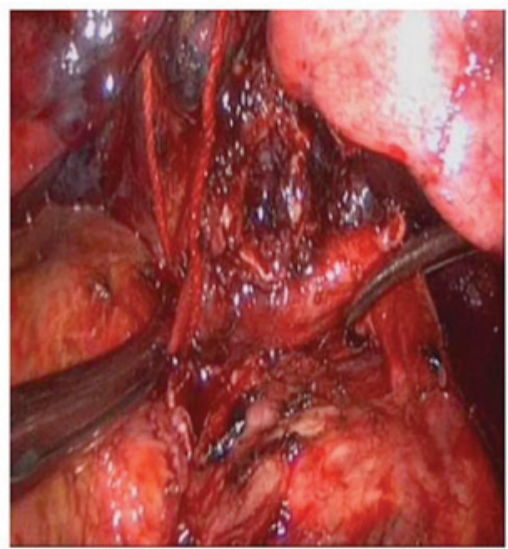

D

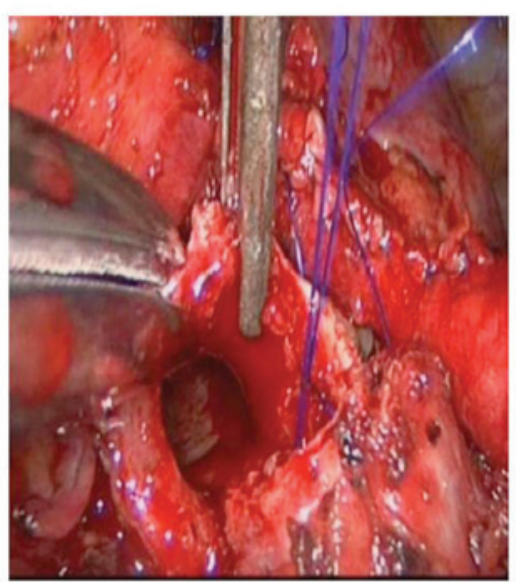

Figure 2. Images captured of the VATS bronchial sleeve resection and pulmonary angioplasty. (A) The superior pulmonary vein was resected using a 45-mm white linear stapler. (B) The left pulmonary artery, which partly had tumor invasion, was freed and blocked. (C) The left pulmonary artery was repaired using a continuous prolene suture. (D) The anastomosis between the left main bronchus and left lower lobe bronchus were processed using a prolene suture.

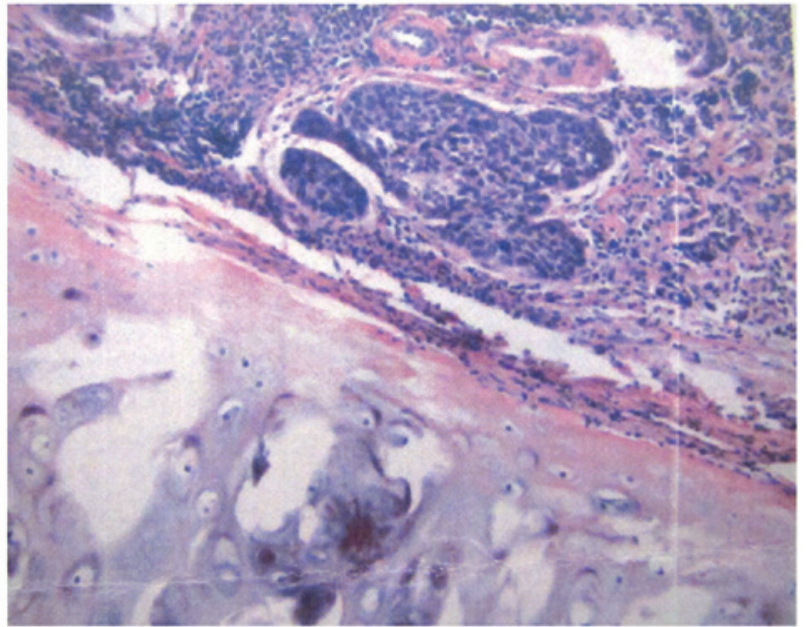

Figure 3. The postoperative pathology revealed that the result of the microscopic examination was squamous cell carcinoma.

\section{Discussion}

It is now well established that, with the improvements in technique and instrumentation, VATS has become a replacement method of choice for a number of procedures that were formerly carried out via thoracotomy (1). The advantages of
VATS for lobectomy have been previously reported, including reduced perioperative pain, shorter hospital stays, a more rapid resumption of normal daily activities, less impairment of pulmonary and shoulder function, reduced rates of cytokine release, and an improved delivery of adjuvant chemotherapy (2). Central bronchogenic carcinoma often invades the bronchus and pulmonary artery, and sleeve lobectomy with pulmonary arterioplasty or bronchoplasty were traditionally performed with a posterolateral thoracotomy (3). Formerly, sleeve resection has been viewed as a contraindication to VATS lobectomy. However, recently VATS sleeve lobectomy was considered as the preferred minimally invasive method in view of its higher operation safety, lower complications, decreased pain and improved quality of life, particularly when the patients presented with long-term smoking, chronic obstructive pulmonary disease and poor pulmonary function, as previously described (4).

Han et al $(5,6)$ hypothesized that, compared with the open surgery, the VATS procedure would generally be likely to be more technically challenging for the transmission from the direct view to a local two-dimensional screen, and from multi-angle, multi-direction operational field to one directional operation field. Therefore, in comparative terms, a markedly clearer anatomical view of the travel, distribution and variation of the pulmonary vessels was required. In addition, others have argued that, in VATS lobectomy, when all the five lobes 
are considered, the biggest difficulty of the operation resides with a VATS left upper lobectomy due to the deep location, special operation angle and predominant vessel invasion of the tumor, particularly central-type pulmonary tumors (7). However, as with all other procedures, VATS sleeve lobectomy has had its own learning curve for surgeons. With the accumulation of experience, continuous suture has been able to be used to complete both membranous bronchus and cartilage anastomosis at one time through thoractomy. Consequently, surgeons have been able to perform continuous suture to complete end-to-end anastomosis using VATS, and to avoid tangling the untied ends.

Additionally, it was our opinion that the left pulmonary artery could more easily be prone to tumor invasion since the left pulmonary artery trunk is shorter, although it has more branches with more fissions and cross-sections. The left pulmonary artery is not shielded by other vessels lying ahead of it, but the right pulmonary artery is shielded by the superior vena cava. In comparison with the right pulmonary artery, the left pulmonary artery is more readily exposed to invasion by the central type pulmonary carcinoma in the left upper lobe. In order to reserve more pulmonary tissue, it was necessary to perform a pulmonary angioplasty. Routinely, pulmonary arterioplasty comprises four operational modes, namely, direct suture, pericardial patch angioplasty, end-to-end anastomosis and pericardial tube replacement (8). From the technical perspective, direct suture appeared to be the most feasible to perform. However, the principle of a successful direct suture is to guarantee that there are no obstructions, and no stenosis for the distal section of the artery; otherwise, pericardial patch angioplasty or end-to-end anastomosis ought to be performed instead (9). However, it was our opinion that, courtesy of the improvements in operational proficiency and surgical instruments, the other three operational modes are also able to be completed using VATS.

The success of totally VATS bronchial sleeve lobectomy and pulmonary angioplasty for the left upper lobe would offer more central type lung carcinoma patients the opportunity to accept the minimally invasive operation, and expand the operation indication of totally VATS lobectomy. Our hope is that total VATS bronchial sleeve and pulmonary angioplasty lobectomy will obtain a more widespread application for the surgical treatment of central type lung carcinoma in the future.

Two major conclusions may be drawn from the present case report: i) Simultaneous end-to-end anastomosis of bronchus and pulmonary angioplasty may be completed using VATS. This indicates that VATS is applicable for the treatment of central bronchogenic carcinoma with invasion of the main bronchus and pulmonary artery; and ii) with the improvements in operational proficiency and surgical instruments, more complicated surgical procedures may also be completed using VATS; therefore, the surgical indications are that the applicability of VATS lobectomy as a technique will be expanded in the future.

\section{References}

1. Schmid T, Augustin F, Kainz G, Pratschke J and Bodner J: Hybrid video-assisted thoracic surgery-robotic minimally invasive right upper lobe sleeve lobectomy. Ann Thorac Surg 91: 1961-1965, 2011.

2. Yamashita Y, Harada $\mathrm{H}$ and Misumi K: Video-assisted thoracic surgery lobectomy for lung cancer: The point at issue. Gen Thorac Cardiovasc Surg 59: 164-168, 2011.

3. Nakanishi R, Fujino Y, Oka S and Odate S: Video-assisted thoracic surgery involving major pulmonary resection for central tumors. Surg Endosc 24: 161-169, 2010.

4. Predina JD, Kunkala M, Aliperti LA, Singhal AK and Singhal S: Sleeve lobectomy: Current indications and future directions. Ann Thorac Cardiovasc Surg 16: 310-318, 2010.

5. Han Y, Zhou S, Yu D, Song X and Liu Z: Video-assisted left upper bronchial sleeve lobectomy. J Thorac Dis 5 (Suppl 3): S304-S631, 2013.

6. Han Y, Zhou S, Yu D, Song X and Liu Z: Video-assisted thoracic surgery (VATS) left upper sleeve lobectomy with partial pulmonary artery resection. J Thorac Dis 5 (Suppl 3): S301-S331, 2013.

7. Gonzalez-Rivas D, de la Torre M, Fernandez R and Mosquera VX: Single-port video- assisted thoracoscopic left upper lobectomy. Interact Cardiovasc Thorac Surg 13: 539-541, 2011.

8. de Leyn P and Decker G: Surgical treatment of non-small cell lung cancer. Rev Mal Respir 21 (5 Pt 1): 971-982, 2004.

9. Okada M, Tsubota N, Yoshimura M, Miyamoto Y, Matsuoka H, Satake S and Yamagishi H: Extended sleeve lobectomy for lung cancer: The avoidance of pneumonectomy. J Thorac Cardiovasc Surg 118: 710-714, 1999. 